\title{
Numerical Simulation of Atomization in Nozzle Injection Flow"
}

\author{
Qinyin FAN**, Chenhai GUO**, Tosimi TAKAGI***, Kikuo NARUMIYA*** \\ and Hiroshi HATTORI*** \\ **Jiangsu University \\ 301 Xuefu Road, Zhenjiang, Jiangsu, 212013, China \\ E-mail: fqyyhoo@yahoo.co.jp \\ *** Osaka Sangyo University \\ 3-1-1, Nakagaito, Daito, Osaka, 574-0013, Japan
}

\begin{abstract}
At the initial stage of injection, the injection flow has not yet broken up and in a range of small atmosphere pressure $(16 \sim 500 \mathrm{KPa})$, the tip of the injection flow always forms a shape of mushroom. ${ }^{[1][2]}$ Moreover, the umbrella of the mushroom is always very big and its root is always very thin, especially when the atmosphere pressure is relatively low $(88 \mathrm{KPa}$, or $100 \mathrm{mmHg})$. These phenomena are not known popularly and the reason of mushroom formation is not clear. In this paper, with the MARS method for simulating free surface, analysis of injection flow is practiced. The phenomena are reproduced and the reason is cleared that the formation of the mushroom is induced by the momentum exchange between the injection fuel flow with very high speed and the very complex flow of the air.
\end{abstract}

Key words: Injection Flow, Free Surface, MARS, Spray, Diesel Engine

\section{Introduction}

In combustion chamber of self-ignition engines, the characteristics of injected fuel flow will dominantly affect the process of ignition, the process of combustion and the emission amount of pollution. Considering the necessary time to form mixture in a practical engine, the detail analysis of the spray developing process and the mechanism of the atomization in the initial stage just after the injection from the nozzle will be most significant. However, in experiments, the measurement is more than extremely difficult for the transient spray with rather high pressure and rather high speed. In numerical analysis, how to define the computational domain and fix the suitable boundary conditions for the domain are always not easy. Few analyses of time-oriented particulars have been reported about the developing process of the injection flow right from the beginning when the flow is injected from the nozzle. In spite of that the nozzle injection process of the high pressure compressed fuel into atmosphere gas with also high pressure has been reported ever so much, the mechanism of the breakup of the injection flow has not yet been explained clearly and has not become a distinct guideline. Investigating the characteristics of the injection flow with both continuous shooting and single shot, authors performed a bunch of observation experiments focusing on magnification technique of injection flow. At the initial stage of injection, the injected flow has not yet broken up and in a range of small atmosphere pressure $(16 \sim 500 \mathrm{KPa})$, the tip of the injected flow always forms a shape of mushroom. Moreover, the umbrella of the mushroom is always very big and its root is always very thin, especially when the atmosphere pressure is relatively low $(88 \mathrm{KPa}$, or $100 \mathrm{mmHg})$. These phenomena are not known popularly and the reason of mushroom formation is not clear. 
In this paper, with the MARS ${ }^{[3]^{[4]}[5]}$ method for simulating free surface, analysis of injecting flow is practiced. The phenomena are reproduced and the reason is cleared that the formation of the mushroom is induced by the complex momentum exchange between the fuel flow with very high speed and the involved flow of the air. In addition, we also find the mushroom shape does not appear at low ambient pressure of $16 \mathrm{KPa}$ in the calculations, which agrees the experiments well.

\section{MARS Method}

In the paper, an accurate algorithm for interface advection and reconstruction, called

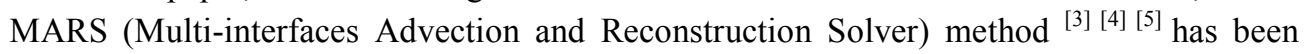
used, which is one of the useful methods for simulating free surface. That is to say, the MARS method is one of the methods, which can simulate multiple phase flow with free surfaces.

The MARS for solving general multiphase flow problems with many free surfaces and interfaces was developed by Kunugi ${ }^{[3]}{ }^{[6]}$. The MARS is a direct numerical simulation (DNS) and it consists of the continuum surface force model (CSF model ${ }^{[5]}$ ) for the surface tension as a volumetric force in the momentum equation and a piece-wise linear interface calculation (PLIC ${ }^{[7]}$ ) for reconstructing the interface between neighborhood computational cells. The MARS meets these important features and includes an interfacial shear stress for the coupling of two fluids (such as gas and liquid) at the interface. However, the discontinuity of the surface reconstruction at the cell boundary was still observed even if the time increment was smaller than the CFL (Courant-Friedrichs-Lewy) condition. In order to improve the reconstruction procedure, a fractional step algorithm was introduced into the fluid-fraction transport equation ${ }^{[8]}$. Resulting from this improvement, more accurate transport of a complicated rigid body has been done.

Although the MARS method is basically the same as VOF (Volume of Fluid) method ${ }^{[9]}$, the MARS method has the following 3 points, which can feature the method. The method 1) introduces CSF surface tension model, 2) considers the gradient of the boundary surface between fuel and gas, and 3) uses an algorithm on the boundary surface, which considers the continuousness of the value of VOF on the boundary surface. Please refer the detail of all these in related papers ${ }^{[3]}{ }^{[4]}[5]$.

The governing equations are mass conservation equation,

$$
\frac{\partial\langle V O F\rangle}{\partial t}+\sum \frac{\partial\left\langle V O F_{(n)} u_{j}\right\rangle}{\partial x_{j}}=0
$$

momentum conservation equation,

$$
\frac{\partial u_{i}}{\partial t}+\frac{\partial u_{j} u_{i}}{\partial x_{j}}=-\frac{1}{\rho} \frac{\partial p}{\partial x_{i}}+\frac{\partial}{\partial x_{j}}\left[\frac{\langle\langle\mu\rangle\rangle}{\langle\langle\rho\rangle\rangle}\left(\frac{\partial u_{i}}{\partial x_{j}}+\frac{\partial u_{j}}{\partial x_{i}}\right)\right]+g_{i}+\frac{1}{\langle\rho\rangle} F_{v}
$$

Here, $x_{i}$ is coordinate, $u_{i}$ is velocity in $x_{i}$ direction, $t$ is time, $\rho$ is density, $p$ is pressure, $\mu$ is viscosity, $g_{i}$ is gravity, $F_{V}$ is volume force on fluid surface, $V O F_{(n)}$ is volume fraction of $\mathrm{n}$-th fluid, $<>$ is averaged properties based on volume fraction. And,

$$
\begin{aligned}
& \langle\rho\rangle=\sum_{n=1}^{2}\left(V O F_{n} \rho_{n}\right)=V O F_{1} \rho_{1}+V O F_{2} \rho_{2} \\
& \langle\langle\varphi\rangle\rangle=\frac{1}{2}\left[\varphi_{1} \times\left\{\operatorname{sign}\left(V O F_{1}-V O F_{2}\right)+1\right\}+\varphi_{2} \times\left\{1-\operatorname{sign}\left(V O F_{1}-V O F_{2}\right)\right\}\right]
\end{aligned}
$$

Following, the continuum surface tension model, advection of boundary surface considering interface slope and reconstruction algorithm for interface advection are generally overviewed.

The general form of the surface tension can be expressed as: 


$$
\left(p_{1}-p_{2}+\sigma \kappa\right) \hat{n}_{i}=\left(\tau_{1 i k}-\tau_{2 i k}\right) \hat{n}_{k}+\frac{\partial \sigma}{\partial x_{i}} \quad, \quad \hat{n}_{i}=\frac{n_{i}}{|n|}=\frac{\partial V O F / \partial x_{i}}{|n|}
$$

Here, $p$ is pressure of fluid, subscripts 1, 2 are fluid number, $\sigma$ is surface tension coefficient, $\tau_{m, i k}$ is viscous shear stress, $n$ is unit normal vector of the interface towards fluid 2, VOF is volume fraction of fluid, and subscript $\mathrm{i}$ or $\mathrm{k}=1,2,3$.

The notation $\kappa$ denotes curvature of a free surface and is defined as:

$$
\kappa=-\frac{\partial \hat{n}_{i}}{\partial x_{i}}=\frac{1}{|n|}\left[\frac{n_{i}}{|n|} \frac{\partial|n|}{\partial x_{i}}-\frac{\partial n_{i}}{\partial x_{i}}\right]
$$

Now, suppose that a fluid to be analyzed is an inviscid for ease and that a surface tension coefficient does not vary along the free surface. Since pressure difference on the free surface is expressed as $p_{2}-p_{1}=\sigma \kappa$, the surface tension force $F_{s}\left(x_{s}\right)$ per unit area acting at a point $x_{s}$ on the free surface can be represented as
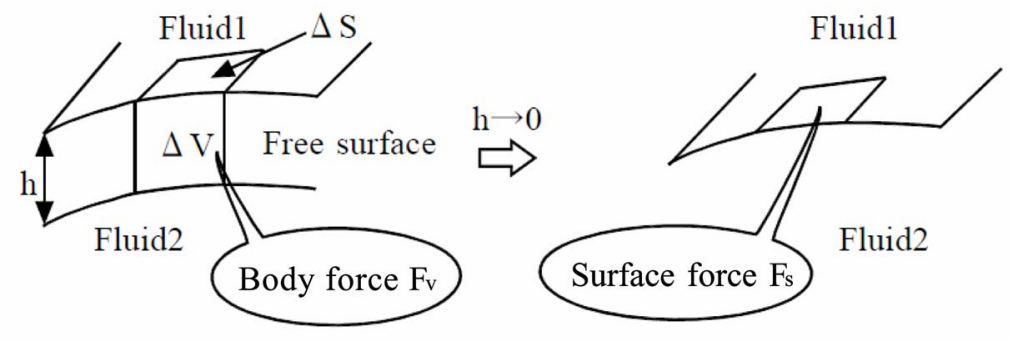

$$
F_{s}\left(x_{s}\right)=\sigma \kappa\left(x_{s}\right) \hat{n}\left(x_{s}\right)
$$

Fig. 1 Image of CSF model ${ }^{[7]}$

In the paper, the continuum surface force (CSF) model ${ }^{[7]}$ is adopted to represent an effect of the surface tension force and to replace a surface force by a body force, the former of which cannot adequately be included in the momentum equation as it is. As shown in Figure 1, imagine that a body force acting on a unit volume surrounding a free surface is compressed in a direction normal to the free surface. Then, the body force will coincide with the surface force acting on a unit area of the free surface.

This idea can specifically be described as follows:

$$
\lim _{h \rightarrow 0} \int_{\Delta v} F_{V}(x) d V=\int_{\Delta S} F_{S}\left(x_{S}\right) d S
$$

By substituting equation (3) for the right-hand side of equation (4), the surface force can be expressed by the body force based on the CSF model as:

$$
F_{v}(x)=\sigma \kappa(x) n(x) \frac{\rho(x)}{\bar{p}}, \quad \bar{p}=\frac{\rho_{1}+\rho_{2}}{2}
$$

The VOF method ${ }^{[10]}$ could capture shapes of interfaces by introducing volume fraction of fluid (VOF value) and by transporting the VOF value to a neighboring fluid element based on a fluid velocity and distribution of the VOF value on each element. However, the most serious factor in causing such results is that interfaces dealt with by the VOF method are assumed to be parallel to either one of coordinate axes. In other words, it can also be said that a slope of the interface crossing neighboring two elements is completely ignored. The MARS method, to the contrary, overcomes those deficiencies of the VOF method through the following procedure. Considering a shape of an interface within an element shown on left side of Figure 2, we calculate the $\delta V O F$ to be transported to a neighboring element during a unit time step $\delta t . S(x)$ being defined as an area occupied by the fluid 1 on a boundary between the notable and the neighboring elements and $u$ as a velocity component in a direction normal to the boundary, $\delta V O F$ can be expressed as follows: 


$$
\delta V O F=\int_{x-u \delta t}^{x} S(x) d x
$$

To consider the slope of the interface for the advection of the VOF value means how the $S(x)$ can be estimated with high accuracy. In other words, a problem about the slope of the interface can be changed for a problem how to calculate the $S(x)$. Now, introduce a continuous linear function $C(x)$ (hereinafter referred to as line-segment function) in calculating the $S(x)$ in equation (6). When considering an element in which a curvature effect on a simplified shape of an interface illustrated on left side in Figure 2 is negligibly small, this line-segment function $C(x)$ can be defined as a function to approximate the $S(x)$ distribution. Accordingly, if $C(x)$ can be given in some way, $\delta V O F$ can analytically be evaluated through equation (6) and the relation $C(x)$. Let us calculate the line-segment function $C(x)$ next. In determining $C(x)$, a size of an element is normalized by each element width, $\Delta x$ and $\Delta y$, as shown on right side in Figure 2. In addition, suppose the $X$ coordinate to calculate the slope of the interface being $x_{0}$. Here, although $x_{0}$ is in agreement with a center of the normalized element, $x_{0}$ is regarded as unknown at the present time. A gradient of $C(x)$ can be represented by the slope $n_{x}$ of the interface at the point $x_{0}$, that is, normal vector on the free surface.

Therefore, $C(x)$ becomes

$$
\left.C(x)=n_{x} x+\frac{1}{2}, \quad n_{x}=\frac{\partial V O F}{\partial x}\right]_{x_{0}}
$$

Note that $C(x)$ is decided so as to be equal to 0.5 at $x=x_{0}=0$. Meanwhile, $C(x)$ denotes a function on the non-dimensionalized coordinate system, whereas what is needed for us is to obtain $S(x)$ itself. Fortunately, $C(x)$ being the function capable of approximating $C(x)$ with enough accuracy, $S(x)$ becomes

$$
S(x) \approx n_{x}\left(x_{0}+x\right)+\frac{1}{2}
$$
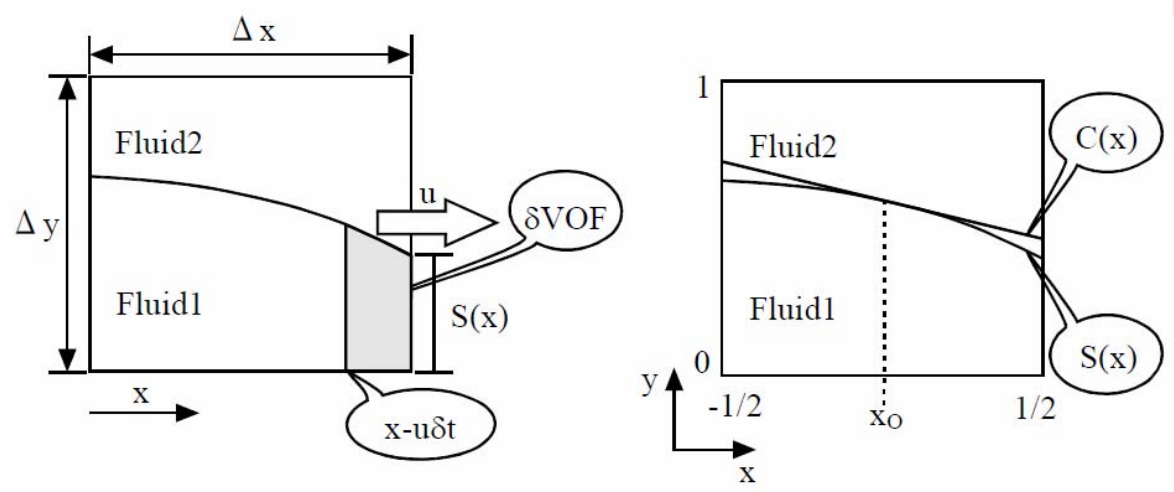

Fig. 2 Approximation via linear function for shape surface

Consequently, what is needed to be solved is the coordinate $x_{0}$ alone. The volume fraction $\delta V O F$ defined by equation (6) to be advected during a unit time interval can be estimated analytically as an area of a trapezoid surrounded by $C(x)$ and grid lines through a relation among equations (7) and (8) and on right side of Figure $2 .{ }^{[8]}$ To sum up, the MARS method is able to determine the area $S(x)$ on the element face analytically by using the line-segment function which represents a linear function of the shape of the free surface via the normal vector of the free surface, and hence is capable of achieving highly-accurate advection of the volume fraction of fluid in comparison with the conventional VOF method.

At last, we briefly explain a reconstruction algorithm necessary for advecting free surfaces. The advection of the free surfaces is carried out by solving the following advection equation for the VOF variable as the VOF method does: 


$$
\frac{\partial V O F}{\partial t}+\frac{\partial V O F \cdot u_{i}}{\partial x_{i}}-V O F \frac{\partial u_{i}}{\partial x_{i}}=0
$$

Equation (9) consists of a time-derivative and an advection terms. In generally dealing with the advection term in equation (9), a kind of interpolation function such as a higher-order advection scheme or CIP (Cubic Interpolation Profile) method is introduced to remove an influence on numerical diffusion. As a result, accuracy of capturing interface via equation (9) is improved, though an alternative approach is adopted in MARS method. Now, we focusing on the second term on the left-hand side of equation (9), a value of VOF- $u_{i}$ corresponds with the itself defined by equation (6). Therefore, equation (9) is discretized as follows:

$$
\begin{aligned}
& \frac{V O F^{n+1}-V O F^{n}}{\Delta t}+\frac{\partial V O F_{i+1}^{n+1}-\partial V O F_{i}^{n+1}}{\Delta x}+\frac{\partial V O F_{j+1}^{n+1}-\partial V O F_{j}^{n+1}}{\Delta y} \\
& -V O F^{n} \cdot\left(\frac{u_{i+1}^{n+1}-u_{i}^{n+1}}{\Delta x}+\frac{v_{j+1}^{n+1}-v_{j}^{n+1}}{\Delta y}\right)
\end{aligned}
$$

Or,

$$
\begin{aligned}
& V O F^{n+1}=V O F^{n}-\Delta t\left(\frac{\partial V O F_{i+1}^{n+1}-\partial V O F_{i}^{n+1}}{\Delta x}+\frac{\partial V O F_{j+1}^{n+1}-\partial V O F_{j}^{n+1}}{\Delta y}\right) \\
& +\Delta t \cdot V O F^{n} \cdot\left(\frac{u_{i+1}^{n+1}-u_{i}^{n+1}}{\Delta x}+\frac{v_{j+1}^{n+1}-v_{j}^{n+1}}{\Delta y}\right)
\end{aligned}
$$

Here, superscript $n$ is present time, superscript $n+1$ is time to be evaluated, $\Delta x$ and $\Delta y$ are mesh width in $X$-direction and $Y$-direction respectively. In order to improve the prediction accuracy of capturing the free surfaces in the MARS method, the calculation of equation (10b) is repeated $\mathrm{N}$ times during a unit time interval $\Delta t$. This method is allowed to compensate various kinds of information about the VOF value on each element and to minimize difference of the VOF values which appears on a boundary between neighboring elements. That is to say, by redefining $\Delta t$ as $\Delta t / \mathrm{N}$ in equation (10b), the calculation of equation (10b) is repeated $\mathrm{N}$ times. In this paper $\mathrm{N}=5$.

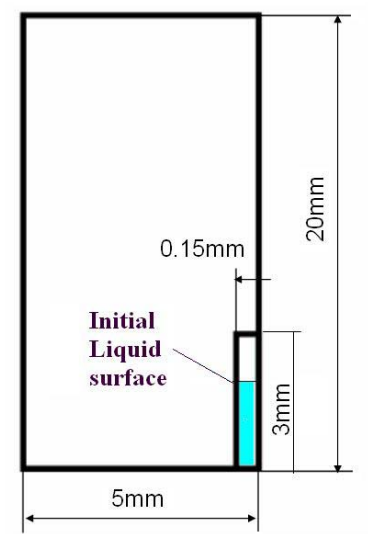

Fig. 3 Calculation domain and nozzle position

\section{Calculation Conditions}

The domain is shown in Figure 3 and it is $5 \mathrm{~mm}$ in radius direction and $20 \mathrm{~mm}$ in axial direction. The left and bottom boundaries of the domain are no-slip walls and the right boundary of the domain is the axial symmetrical centerline. The upper boundary of the domain is outlet where natural in-out boundary condition (zero gradient of pressure) is defined. The domain is divided into 360,000 grids (300 grids uniform in radius direction and 1200 uniform in axial direction). 
Fuel is injected from a nozzle. The diameter of the nozzle is $0.3 \mathrm{~mm}$ and the length of the nozzle is $3 \mathrm{~mm}$. The axis of the nozzle is arranged along the axial symmetrical centerline and the initial surface of the fuel is defined at a plane that is $2 \mathrm{~mm}$ from the inlet of the nozzle (Fig. 3). That is to say the fuel flow will be injected out after flowing $1 \mathrm{~mm}$ in the nozzle. It is of great significance in this simulation to consider the effects of the fuel flow behavior in the nozzle to the flow patterns out of the nozzle.

Table 1. Calculation Conditions of Cases

\begin{tabular}{|c|c|c|}
\hline Case & Atmosphere Pressure $(\mathrm{Pa})$ & Air Density $\left(\mathrm{kg} / \mathrm{m}^{3}\right)$ \\
\hline A & 16000 & 0.19027 \\
\hline A2 & 48000 & 0.57081 \\
\hline A3 & 61000 & 0.72540 \\
\hline A4 & 75000 & 0.89189 \\
\hline B & 88000 & 1.06450 \\
\hline C & 101300 & 1.20500 \\
\hline D & 500000 & 5.00700 \\
\hline
\end{tabular}

SI unit is used in the paper. The temperature of atmosphere is $293 \mathrm{~K}$. Because of the little effect of pressure, the properties of light fuel at $293 \mathrm{~K}$ are defined as constants, which do not vary with the change of pressure. They are density $\rho_{0}=840 \mathrm{~kg} / \mathrm{m}^{3}$, viscosity $\mu_{0}=2.0 \times 10^{-3} \mathrm{Ns} / \mathrm{m}^{2}$, surface tension $\sigma_{0}=20 \times 10^{-3} \mathrm{~N} / \mathrm{m}$. The properties of air are viscosity $\mu_{\text {air }}=17.98 \times 10^{-6} \mathrm{Ns} / \mathrm{m}^{2}$, surface tension $\sigma_{\text {air }}=0 \mathrm{~N} / \mathrm{m}$. They are also constants, which do not vary to pressure change. And the density of air $\rho_{\text {air }}$ is calculated by the equation 11 , which varies with pressure. The atmosphere pressure and density in various cases are listed in Table 1 .

$$
\rho=\frac{1}{0.287 \times 10^{3}} \frac{p}{T}
$$

Where $p$ is pressure, $T$ is temperature of air, and $\rho$ is the density of air. All calculations are transient ones with constant time steps of $1.25 \times 10^{-8} \mathrm{~s}$. The end of calculation time is $75 \mu$ s for all cases. The injection pressure is fixed as a constant as shown in Table 1 . When fuel mass fraction in one cell (grid) of the domain is less than $1 \times 10^{-5}$, the program will consider fuel liquid mass fraction as zero in the cell.

\section{Results of Calculations Compared with Experiments}

In the very initial stage of the diesel engine injection flow experiments ${ }^{[1]}{ }^{[2]}$, when the injection fuel flow has not yet broken up, the tip of the injection flow shapes a mushroom. And the umbrella becomes big and the stem becomes very long and thin. In a region of low atmosphere pressure about $50 \sim 100 \mathrm{KPa}$, it goes a little overboard. When the atmosphere pressure becomes much lower, for example, $16 \mathrm{KPa}$, the mushroom shape of the flow does not appear in the injection after all. It should be noted that the flow injected from the nozzle becomes spray directly when it goes out of the nozzle in the case of $16 \mathrm{KPa}$. The above phenomena are not known widely by researchers and engineers. The various shapes of the injecting flow in different atmosphere pressures are plotted in Figure 4, where the injecting pressure is $17 \mathrm{MPa}$ in all cases and the pressures of atmosphere ambient in the cases are respectively $88,75,48$ and $16 \mathrm{KPa}$ from left to right. 


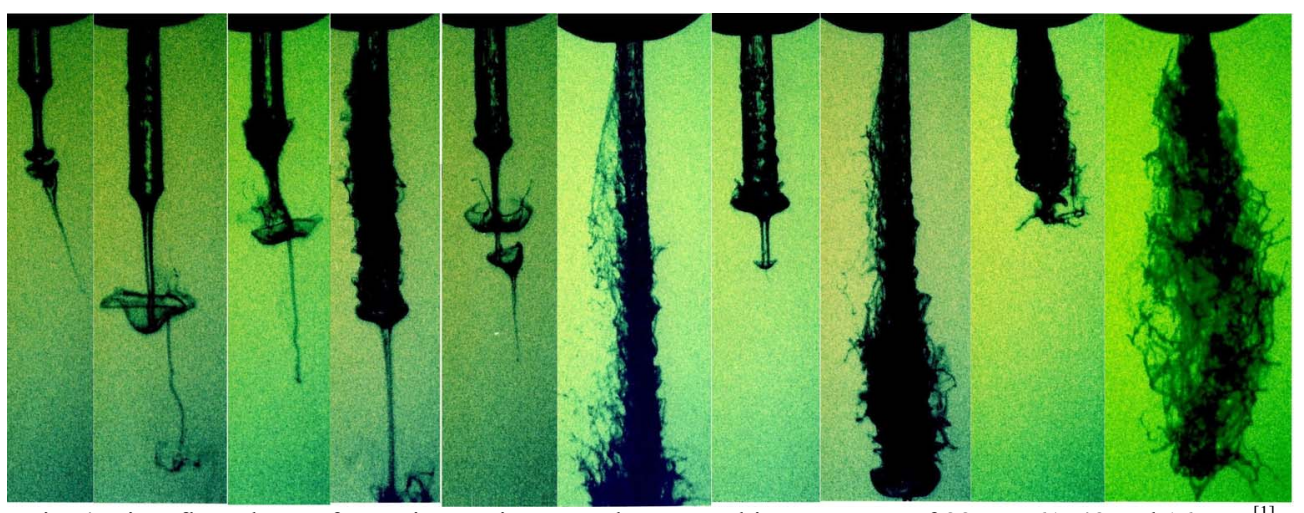

Fig. 4 Inject flow shape of experiments in atmosphere at ambient pressure of $88,75,61,48$ and $16 \mathrm{KPa}^{[1]}$

$$
\begin{array}{ll}
\text { Cycle: } & 6000 \\
\text { Time : } & 0.000075
\end{array}
$$
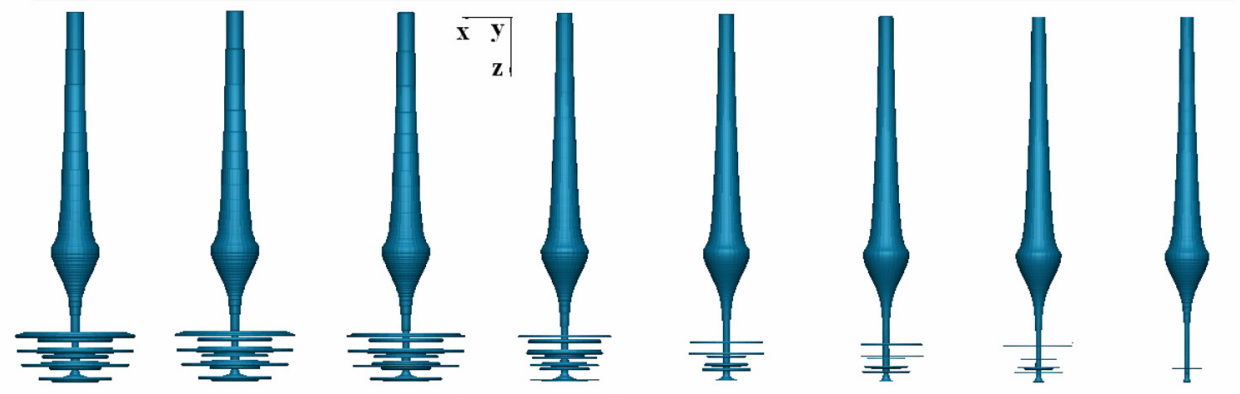

Fig. 5 Results of Case B of calculations at ambient pressure of $88 \mathrm{Kpa}$

No speculation has taken place concerning why the injection flow becomes the mushroom shape. And with only experiment data, it is even difficult to understand why the mushroom shape is induced and how to explain the behavior and the mechanism of the injection flow. Authors suggest that simulation will help us to describe the injection flow with details and help us to understand the behavior and the mechanism. The MARS method is selected due to that it can simulate multiple phases flow with free surfaces ${ }^{[3]}{ }^{[4]}{ }^{[5]}$.

In Figure 5, the calculated results of Case B in Table 1 are shown. The atmosphere pressure of Case B is similar with the most left case of experiments in Figure 4. The atmosphere pressure is $88 \mathrm{KPa}$ and the injection pressure is $17 \mathrm{MPa}$. From left to right, different fuel mass fraction is plotted for the same Case B, which are 0.0001, 0.001, 0.01, $0.10 .3,0.5,0.7$ and 0.9 . In the figure, plotted with different mass fractions, the inject flow shapes have the same characteristics which appear in experiments. In the tip, a mushroom shape is induced and the umbrella is big. The stem of the injection flow is thin near the nozzle outlet and becomes thicker and thicker later. Near the tip, the stem becomes extremely thin. Although, all these characteristics of both experiments and analyses are similar, the flow shape of different mass fractions greatly changes from fraction 0.0001 to 0.9. Otherwise, in the experiments, the boundary of the inject flow photos has not direct relationship with fuel fraction, where the mass fraction is difficult to know exactly. It should be concluded that what value of the mass fraction in calculations should be chosen to compare with the experiments is not easy to determine and should be discussed in detail.

Based on the comparisons of analyses and experiments, we try to explain the reasons why the mushroom of the inject fuel flow is shaped. In calculations, with an axial symmetrical mesh, the fuel drops broken up from the injected flow will form the circles around the injection tip and further more these circles form an umbrella. The flow in the 
nozzle becomes thinner than the nozzle diameter before the flow goes out of the nozzle due to the friction with the nozzle walls and it becomes thinner when it is compressed by the airflow out of the nozzle.

It is important to notice that 1) the friction between fuel and nozzle wall affects the flow and the flow becomes thinner near the outlet of the nozzle, and the tip of the flow with very high velocity moves slower, 2 ) the air near the flow is accelerated by the injection flow and the air velocity distribution becomes very complex, and 3) the velocity of the fuel tip becomes slower and slower and the fuel chokes up to form a thickest part of the fuel flow. In the left of Figure 6, the contour of air velocity magnitude is plotted. We can find that the radius of injection flow changes greatly near the regions where the air velocity is relatively big. Concretely, near the tip and near the thickest part of the flow, the air has the biggest velocity. In the middle of Figure 6 , the contour of velocity component in $x$ direction is shown. As shown by the color bar, the velocity in a region of $5-10 \mathrm{~m} / \mathrm{s}$ is plotted in dark black. That means the air will flow away from the central axis, or the air will pull the fuel flow away and let it become thicker. In the reverse, in the right of Figure 6, the velocity less than $0(0 \sim-20.0 \mathrm{~m} / \mathrm{s})$ is plotted in dark black. That means the air flows towards the center axis and pushes the fuel flow to the axisymmetrical axis and let it become thinner. We should consider that the relationship between fuel flow radius and direction of $x$ component of velocity is an inevitable corollary. In other words, the effects to each other between air and fuel (with a very complex flow pattern and a very high injection speed) shaped the mushroom, which was very difficult to understand.

In Figure 7, comparison of calculation (upper) and experiments (below) with different scale is shown. Although the thin root and first mushroom are represented in the simulation, the very thin tip progress after the formation of the umbrella has not yet been regenerated. It should be a topic in the research of next step.

In Figure 8, the shape of the injection flow similar to Case B is plotted in two ways. On the left side, a section of the flow is generated and many particles are gathered around the tip of the flow. These particles form many circles around the tip of the flow on the right in the figure, which is axisymmetrically-plotted.

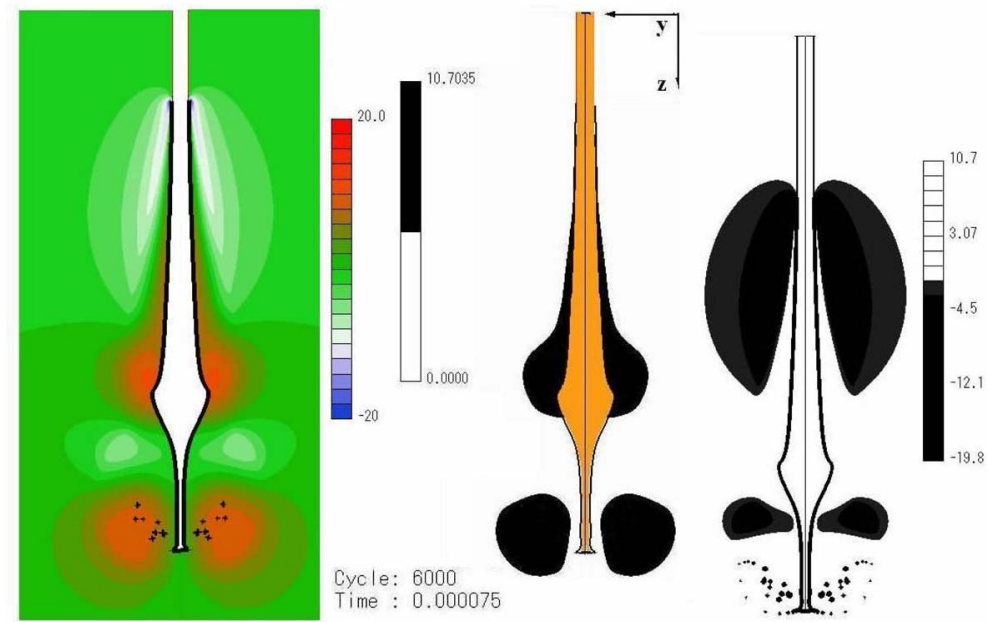

Fig. 6 Contour of velocity magnitude (left), velocity lager than $5 \mathrm{~m} / \mathrm{s}$ in $x$ direction (middle) and velocity less than $0 \mathrm{~m} / \mathrm{s}$ in $x$ direction (right)

Comparing with cases of experiments, when ambient pressure becomes lower and lower from $500 \mathrm{KPa}$ to $16 \mathrm{KPa}$, the injection flow changes its shape (Figure 4 and 9). In detail, at the ambient pressure of $500 \mathrm{KPa}$, the mushroom begins to appear (Figure 9). And the size of the mushroom becomes the biggest at the ambient pressure of $88 \mathrm{KPa}$ and 
becomes smaller after that when the ambient pressure changes to 61 and $48 \mathrm{KPa}$ (Figure 4). At last, the mushroom shape does not appear at the lowest ambient pressure of $16 \mathrm{KPa}$ (the most right of Figure 4) and the flow is atomized completely just out of the nozzle. These facts suggest that at a very low ambient pressure, the injection flow is much easier to atomize than at a very high ambient pressure normally in a diesel engine chamber.

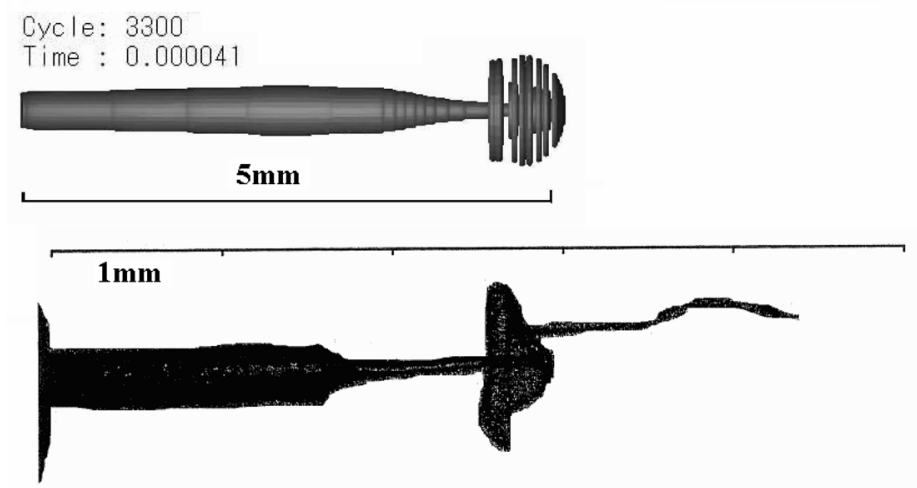

Fig. 7 Comparison of calculation (upper) and experiments (below) with different scale

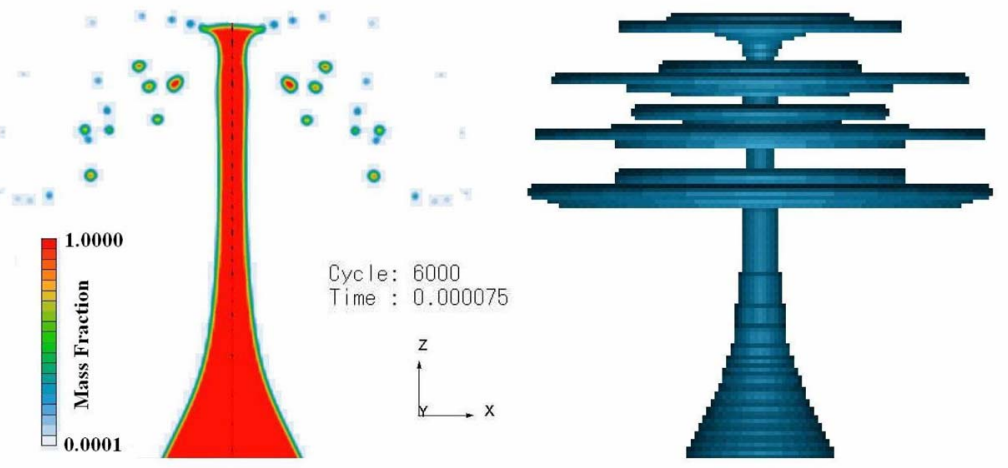

Fig. 8 Injection flow shape of 2D and semi-3D models
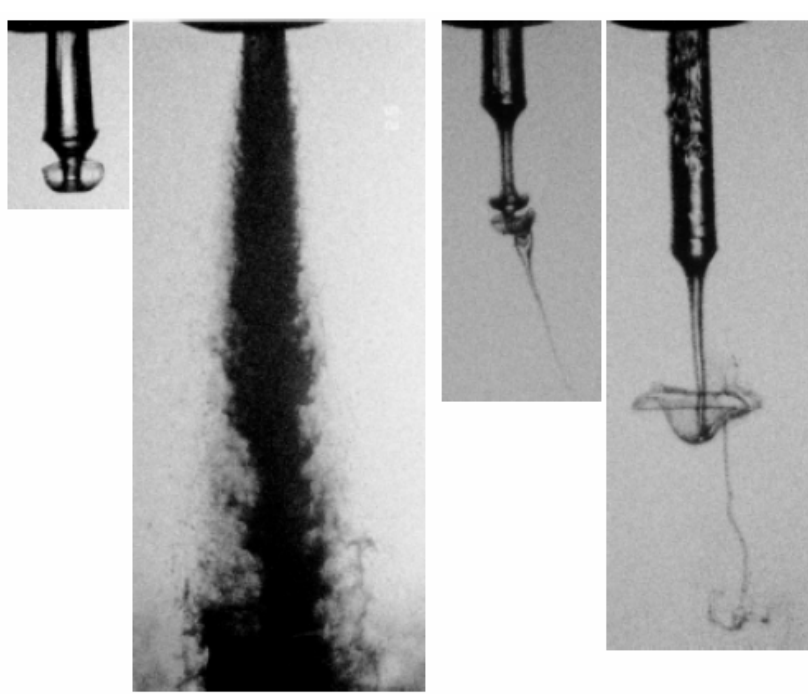

Fig. 9 Shadow graph of mushroom at the injection flow tip in the atmosphere at the ambient pressure of $500 \mathrm{KPa}$ (left) and $88 \mathrm{KPa}$ (right), when inject pressure is $32 \mathrm{MPa}$ (left) and $17 \mathrm{MPa}$ (right) respectively 
The trend of shape change of the injection flow is reproduced in analyses in Figure 10. In calculations, at the ambient pressure of $500 \mathrm{KPa}$, the mushroom shape begins to appear (at bottom of Figure 10). And the size of the mushroom shape becomes the biggest at the ambient pressure of $88 \mathrm{KPa}$ (at 2nd and 3rd line of Figure 10). At last, the mushroom shape does not appear at the lowest ambient pressure of $16 \mathrm{KPa}$ (at top of Figure 10), where the flow is considered to atomize into very dense fuel particles completely out of the nozzle. The trend of shape change is totally similar to the experiments. From this point, we might go on to an even more detailed examination of the multiple umbrella shape of the injection flow. In the experiments, at the ambient pressure of $48 \sim 88 \mathrm{KPa}$, double umbrellas at the tip of the injection flow are observed. Because the tip is very thin in this case, we could suppose that the thin stem between the two umbrellas might be much easier to be reproduced at pressure of $61 \mathrm{KPa}$. Authors are planning to check it carefully.

Ambient pressure affects the properties of fuel, especially the density of ambient air. In this paper, it is cleared completely that the density of air (the ambient pressure) is extremely important to form the mushroom injection flow. Others such as injection pressure and the inner shape of the nozzle are all also important factors affecting the results.

\section{Conclusions}

In this paper, with the MARS ${ }^{[3]^{[4]}[5]}$ method for simulating free surface, analysis of injecting flow is practiced. The phenomena are reproduced and the reason is cleared that the formation of the mushroom is induced by the complex momentum exchange between the inject fuel flow with very high speed and the involved flow of the air. And authors also find the mushroom shape does not appear at low ambient pressure of $16 \mathrm{KPa}$ in the calculations, which agrees to the experiments well. With a reasonable explanation, authors also present the reason of the phenomenon that the injection flow is much easier to atomize at the low ambient pressure than at a very high ambient pressure normally in a engine chamber.

With axial symmetrical meshes, many circles of the fuel liquid form the umbrella part of the injecting flow. As shown in Figure 7, we can image the discrete shape of the umbrella part of the inject flow in a three dimensional mesh, which will be formed by the lumps of many liquid droplets, but not the liquid circles. In other words, the results of three-dimensional simulations will be much closer to the experiments. To verify the imagination, three-dimensional analyses are necessary receiving more accurate results. 
Case $A$ Cycle: 6000 Time: 0.000075

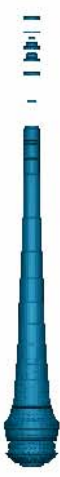

Case $B$ Cycle: 6000 Time : 0.000075
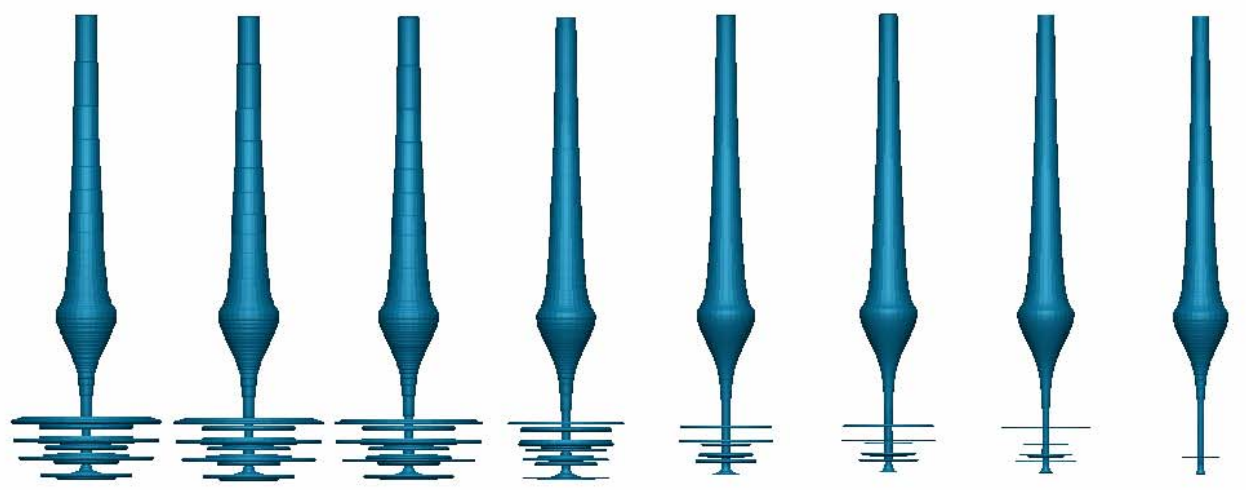

Case $C$ Cycle: 6000 Time : 0.000075
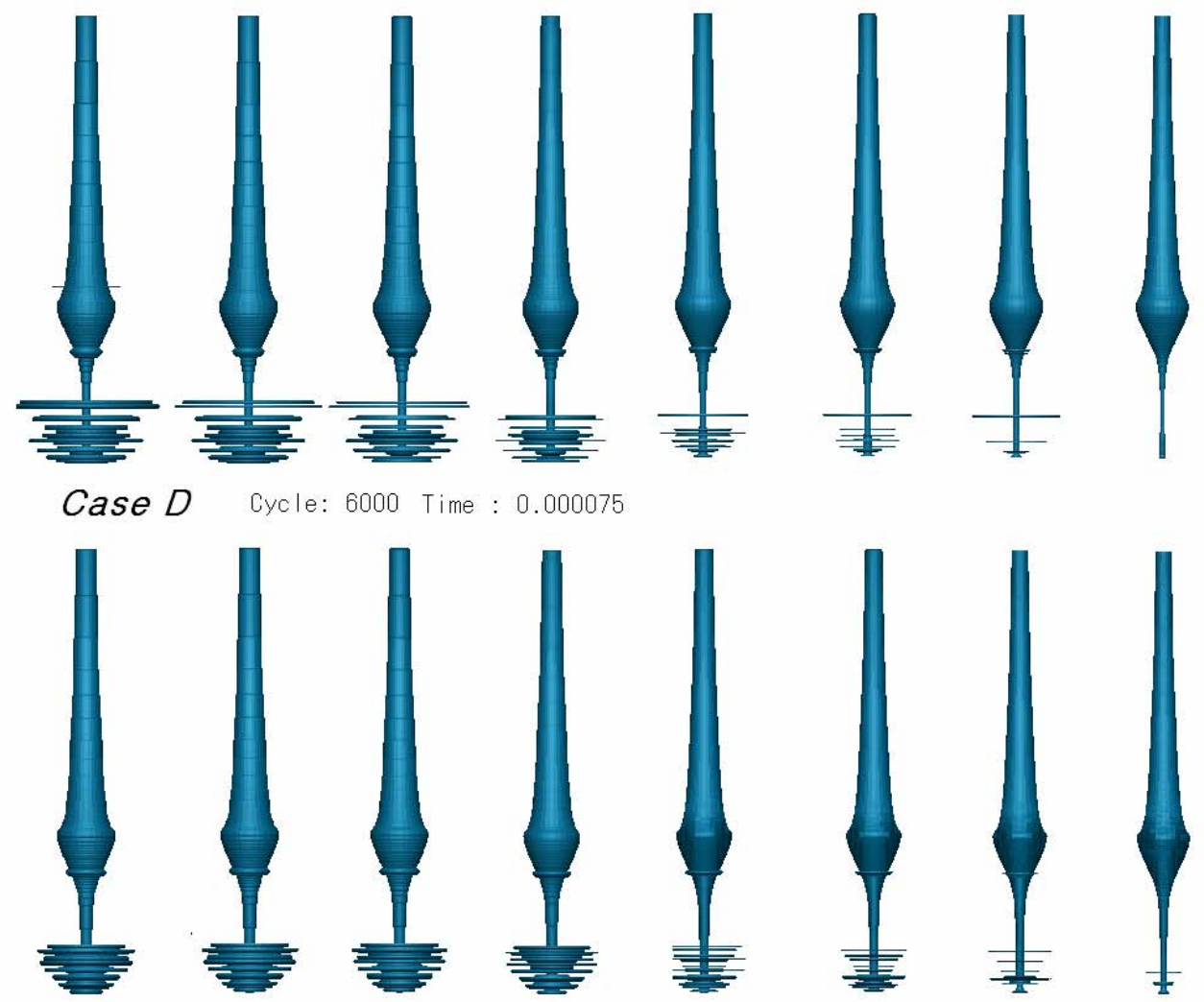

Fig. 10 Shapes of cases in calculations at $0.000075 \mathrm{~s}(75 \mu \mathrm{s})$ when mass fractions are $0.0001,0.001,0.1,0.2$ $0.3,0.5,0.7$ and 0.9 (from left to right in all cases) at the ambient pressure of $16,88,101.3$ and $500 \mathrm{KPa}$ 
On the contrary, there seems to be little agreement quantitatively in the penetration of the inject flow between experiments and calculations. In Figure 7, the penetration of calculation is about 1.5 times bigger than the experiments. The reason could be presupposed as the effects of the different variation of the injection pressure. In the calculations, the injection pressure at the nozzle inlet is defined as a constant value, and in the experiments, the injection pressure always changes from 0 to a maximum value and then vibrates to 0 again ${ }^{[1]}$. Although we have a good reason to believe that the difference of the injection pressure at the nozzle inlet induced the difference of the penetrations, it is not easy to define a rational varying injection pressure according to experimental data or theoretical formula. In other words, a reasonable big domain with boundary, where the conditions can be defined clearly, should be considered. With this model, considering the pressure variation of the fuel in the nozzle cabin, we can expect to estimate more accurate results simulating the penetration and the tip velocity changing.

It should be a topic in the research of next step that the tip progress after the formation of the umbrella in Figure 7 has not been represented in the simulation.

At last, the compressibility of the fuel should be considered in the simulations.

\section{References}

[1] Hiroshi HATORI, Kikuo NARUMIYA, Analysis of Disintegration Process of Diesel Spray (Observation of Spray Tip Behavior by Greatly Magnified Photographs), Report RC116, Society of Automotive Engineers of Japan

[2] H.Hattori, K.Narumiya, M.Tue, T.Kadota, Analysis of Initial Breakup Mechanism of Diesel Spray Injected into High Pressure Ambience, 2004, SAE World Congress 2004-01-0528.

[3] Kunigi, T., Direct Numerical Algorithm for Multiphase Flow with Free Surfaces and Interfaces, Transactions of The Japan Society of Mechanical Engineers, Series B, Vol. 63, No. 609, (1997), pp.1576-1584.

[4] Kunugi, T., Ose, Y. and Banat, M., Slug-plug-flow analyses of stratified flows in a horizontal duct by means of the MARS, 1999, Proceedings of the 5th ASME/JSME Joint Thermal Engineering Conference, San Diego.

[5] Brackbill, J. U., Kothe, D. B. and Zemach, C., A continuum method for modeling surface tension, J. Computational Physics, Vol. 100, (1992), pp.335-354.

[6] Kunugi, T., Direct Numerical Algorithm for Multiphase Flow with Free Surfaces and Interfaces, 1997, Proceeding of ISAC'97 High Performance Computing on Multiphase Flows, pp.25-30.

[7] Youngs, D. L., Numerical Methods for Fluid Dynamics, Ed. Morton, K. W. and Baines, M. J., 1982, Academic Press, pp.273-285.

[8] Kunugi, T. and Ose, Y., Numerical simulation on multiphase flow with phase chang based on MARS method, Proceeding, 1997 Meeting of Japan Society of Fluid Mechanics, Tokyo, Japan. July 29-31, pp. 351-352.

[9] Hirt, C. W. and Nichols, B. D., Volume of fluid (VOF) method for the dynamics of free surfaces, Journal of Computational two-phase flow, 1981, Vol. 39, pp.857-864. 